\title{
A cross-reactive neisserial antigen encoded by the NMB0035 locus shows high sequence conservation but variable surface accessibility
}

\begin{abstract}
Correspondence
Carlos M. Ferreirós

mpcfytc@usc.es
\end{abstract}

Received 19 January 2007

Accepted 13 September 2007

\author{
Jesús Arenas, ${ }^{1}$ Ana Abel, ${ }^{1}$ Sandra Sánchez, ${ }^{1}$ Juan Marzoa, ${ }^{1}$ \\ Sonsoles Berrón, ${ }^{2}$ Peter van der Ley, ${ }^{3}$ María-Teresa Criado ${ }^{1}$ \\ and Carlos M. Ferreirós ${ }^{1}$
${ }^{1}$ Departamento de Microbiología y Parasitología, Facultad de Farmacia, Campus Sur, Universidad de Santiago de Compostela, 15782 Santiago de Compostela, Spain 28220 Majadahonda, Madrid, Spain
${ }^{3}$ Laboratory for Vaccine Research, Netherlands Vaccine Institute, PO Box 457, $3720 \mathrm{AL}$
Bilthoven, The Netherlands \\ ${ }^{2}$ Centro Nacional de Microbiología, Instituto de Salud Carlos III, Ctra. Majadahonda-Pozuelo Km2,
}

\begin{abstract}
The meningococcal NMB0035 locus encodes a $47 \mathrm{kDa}$ outer-membrane protein that is highly conserved antigenically, and is able to induce antibodies during infection and bactericidal responses in vitro. This study analysed the surface exposure of this protein using specific antibodies in flow cytometry assays and determined its nucleotide sequence in 33 Neisseria strains. Genomic analyses revealed no significant differences in the nucleotide or amino acid sequences, but flow cytometry showed that surface accessibility was highly variable among the strains. These results suggest that masking by and/or association with lipo-oligosaccharides or other membrane molecules can be crucial for antigen accessibility, which must be thoroughly analysed in new vaccine candidates.
\end{abstract}

\section{INTRODUCTION}

Current research into vaccine candidates suitable for the prevention of meningitis caused by serogroup B meningococci has focused on outer-membrane proteins (OMPs) and lipo-oligosaccharides (LOSs), either purified or naturally incorporated into outer-membrane vesicles (OMVs) (Morley \& Pollard, 2001). The high antigenic variability of many OMPs limits the efficacy of singleprotein vaccines, and vaccines based on OMVs that contain all of the outer-membrane antigens in their native conformations have failed to induce cross-protection due to the immune dominance of PorA, the serosubtyping antigen, which presents a high degree of variability (Rosenqvist et al., 1995; Tappero et al., 1999; Morley et al., 2001). The prevalence of commensal species as colonizing neisseriae during childhood and the existence of common antigens, particularly between Neisseria meningitidis and Neisseria lactamica, has raised interest in using commensals for vaccination (Gorringe et al., 2005) or as

Abbreviations: LOS, lipo-oligosaccharide; OMP, outer-membrane protein; OMV, outer-membrane vesicle.

The GenBank/EMBL/DDBJ accession numbers for the $p 47$ gene sequences reported in this paper are DQ788636-DQ788662 ( $N$. meningitidis strains) and D0788663-D0788666 (N. lactamica strains). delivery vehicles for meningococcal vaccine antigens (O'Dwyer et al., 2004). Thus, the investigation of new vaccine candidates is using all of the technologies derived from advances in molecular biology (Leclerc, 2003; Khan et al., 2006) and the information obtained from the DNA sequences published for several meningococcal strains. Bioinformatic analyses applied to genetic, molecular and antigenic characteristics of vaccine candidates (Zagursky \& Rusell, 2001; Petrovsky \& Brusic, 2002) can help to determine their genetic divergence, and to predict their structural conformation and antigenic domains (Doytchinova \& Flower, 2002) on the basis of comparison with previously well-characterized molecules registered in molecular databases.

The most important challenge for obtaining a good meningococcal vaccine based on OMPs is to find an antigen or a group of antigens common to all strains that is easily accessible and is able to induce long-term protective immune responses in both adults and children. Knowledge of the variation of many microbial characteristics, particularly surface-antigen expression, has been conditioned by the general assumption that the behaviour of individual bacteria in a population is homogeneous and can be analysed by measuring the population as a whole. Flow cytometry is having a major impact on antigenic analysis, 
and is a valuable technique that allows the simultaneous determination of the accessibility and population variability of surface antigens to specific antibodies (Valdivia \& Falkow, 1998). Previous studies in our laboratory have demonstrated the cross-reactivity of a $47 \mathrm{kDa}$ antigenic neisserial OMP by Western blotting and dot blotting. This antigen is inducible by iron restriction and has been identified by matrix-assisted laser desorption/ionization time-of-flight MS as the product encoded by the NMB0035 (N. meningitidis strain MC58) and NMA0281 (N. meningitidis strain Z2491) loci (Arenas et al., 2006). Experiments with sera from patients convalescing from meningococcal disease (Troncoso et al., 2000) and with a polyvalent specific rabbit serum against this protein (Arenas et al., 2006) have shown that this antigen is able to induce antibodies during infection and bactericidal responses in vitro. To investigate further the vaccine potential of this antigen, which we called P47, this study has extended the previous analysis by using a specific antiserum and flow cytometry techniques to evaluate the accessibility of P47 epitopes on the surface of $27 \mathrm{~N}$. meningitidis and 6 commensal Neisseria strains and, by sequencing the gene in all of them, to establish its conservation.

\section{METHODS}

Bacterial strains and culture conditions. The characteristics of all strains used in this work have been described previously (Arenas et al., 2006). N. meningitidis strains GLD and B16B6 were kindly provided by Dr Borriello (Queens Medical Centre, University Hospital, Nottingham, UK), strain NMB by Dr Granoff (Children's Hospital, Oakland Research Institute, Oakland, CA, USA) and strain M7 (a capsule-deficient mutant of NMB) by Dr Pizza (Immunobiological Research Institute, Siena, Italy). The three strains galE, $r f a C$ and $l p x A$ are different LOS mutants obtained by Dr van der Ley from strain H44/76 (Gorter et al., 2003). All other strains were from our laboratory collection. Multilocus sequence typing (MLST) of each strain was carried out by determination of the allelic profiles obtained from a $450 \mathrm{bp}$ internal region in the $a b c Z, a d k$, aroE, fum, $g d h, p d h C$ and pgm genes. Assignation to clonal complexes was done by comparison of the profiles with those in the meningococcal MLST database (http://pubmlst.org/neisseria/).

Maintenance of the strains and culture under iron-restricted conditions were as described previously (Pintor et al., 1996). For flow cytometry, cells were fixed by adding formaldehyde $(1 \%, \mathrm{v} / \mathrm{v})$ to the culture just after growth and incubating for $30 \mathrm{~min}$ at room temperature with constant shaking at 100 r.p.m. Cells were then washed twice with PBS, suspended at a concentration of $10^{9}$ cells $\mathrm{ml}^{-1}$ in PBS containing $0.05 \mathrm{mM}$ PMSF and maintained at $4{ }^{\circ} \mathrm{C}$ until use. For some experiments, the cells were permeabilized with $70 \%$ ethanol (30 min at room temperature with constant shaking at 100 r.p.m.) prior to treatment with formalin.

Flow cytometry assays. Flow cytometry assays were carried out using a modification of the method described by Welsch et al. (2003) using rabbit sera against both the purified $47 \mathrm{kDa}$ protein and OMVs obtained as described previously (Arenas et al., 2006). Formaldehydefixed bacteria were suspended at a concentration of $10^{7}$ cells $\mathrm{ml}^{-1}$ in $1 \%$ BSA (w/v) in Tris-buffered saline with $0.05 \%$ Tween 20 (TBSA) and blocked for $1 \mathrm{~h}$ at $37{ }^{\circ} \mathrm{C}$ with constant gentle shaking. Aliquots $(1 \mathrm{ml})$ were then stained with BacLight (Molecular Probes) according to the manufacturer's instructions, incubated with working dilutions (1:1000) of the different sera for $90 \mathrm{~min}$ at $37^{\circ} \mathrm{C}$, washed with Trisbuffered saline and finally incubated with Alexa Fluor 488-conjugated goat anti-rabbit IgG (Molecular Probes) in TBSA for $1 \mathrm{~h}$ at $37^{\circ} \mathrm{C}$ with constant gentle shaking. Fluorescence was measured in a Coulter-XL flow cytometer. Bacteria incubated with non-immune rabbit or mouse sera were used to adjust the instrument for non-specific cell-associated fluorescence. Controls using unstained bacteria were included to check for overlapping of fluorescence. For each strain, $10^{4}$ events were recorded and the fluorescence associated with bacterial cells was determined by gating with the BacLight channel values. Fluorescence produced by cells incubated with non-immune serum was used to adjust the counter so that $99 \%$ of cells lay under the $10^{1}$ fluorescence intensity region. Thus, fluorescence intensities reported for immune sera in each experiment were always relative to those obtained using non-immune serum. All experiments were repeated on 2 to 4 different days, and fluorescence intensities reported for each strain were the means of all replicates in all experiments. Data were plotted using WinMDI software (version 2.9; http://www.bio-soft.net/other.html).

Isolation of DNA and PCR amplification. For DNA extraction, cells were suspended in protease- and DNase-free deionized water (approx. $3 \times 10^{8}$ cells $\mathrm{ml}^{-1}$ ) and treated for $20 \mathrm{~min}$ at $100{ }^{\circ} \mathrm{C}$ and then at $-20{ }^{\circ} \mathrm{C}$ for $2 \mathrm{~min}$. The suspension was then centrifuged at $10000 \mathrm{~g}$ for $5 \mathrm{~min}$ and the supernatant recovered and stored in aliquots at $-20{ }^{\circ} \mathrm{C}$ until use.

Detection of the $p 47$ gene was carried out using a primer pair (D-L/D$\mathrm{R}$; Table 1) designed following analysis of the NMA0281 (N. meningitidis Z2491) and NMB0035 (N. meningitidis MC58) loci from public databases (The Institute for Genomic Research, www.tigr.org), which corresponded to an inner fragment of approximately $600 \mathrm{bp}$. PCRs were performed in a MyCycler 1.06 system (Bio-Rad) after optimization of the optimal detection conditions. Each reaction mixture $(20 \mu \mathrm{l})$ contained: $0.5 \mu \mathrm{l}$ DNA solution, $1.5 \mathrm{mM} \mathrm{MgCl}_{2}$, $10 \mu \mathrm{M}$ dNTPs, $0.8 \mu \mathrm{M}$ oligonucleotides, $2 \mathrm{U}$ BioTaq, $16 \mathrm{mM}$ $\left(\mathrm{NH}_{4}\right)_{2} \mathrm{SO}_{4}, 67 \mathrm{mM}$ Tris/HCl $(\mathrm{pH} \mathrm{8.8)}$ and $0.01 \%(\mathrm{v} / \mathrm{v})$ Tween 20. The PCR program comprised pre-incubation at $94{ }^{\circ} \mathrm{C}$ for $5 \mathrm{~min}$, followed by 27 cycles of $94{ }^{\circ} \mathrm{C}$ for $30 \mathrm{~s}, 67^{\circ} \mathrm{C}$ for $30 \mathrm{~s}$ and $72{ }^{\circ} \mathrm{C}$ for $1 \mathrm{~min}$, with a final extension at $72{ }^{\circ} \mathrm{C}$ for $4 \mathrm{~min}$. Positive and negative controls were included in each experiment.

DNA sequencing and phylogenetic analysis. Sequencing of the $p 47$ gene in the different strains was carried out after PCR amplification of five overlapping fragments (Table 1). Primers for amplification (F1 to F5) were designed on the basis of sequence data from the homologous locus NMA0281. Different primer pairs were necessary to amplify fragment 5 . PCR products were purified using a QIAquick gel extraction kit (Qiagen) and the DNA sequenced using a BigDye terminator v3.1 cycle sequencing kit (Applied Biosystems). Each reaction $(10 \mu \mathrm{l})$ contained $4 \mu \mathrm{l}$ purified PCR product, $3 \mu \mathrm{l}$ BigDye terminator RR-100, $1 \mu \mathrm{l}$ primer $(5 \mu \mathrm{M})$ and $2 \mu \mathrm{l} \mathrm{H}_{2} \mathrm{O}$. Amplification and labelling reactions were carried out independently and repeated twice for each chain. Sequencing of fragments was carried out at the Instituto de Salud Carlos III (www.isciii.es) and Sistemas Genomicos S.A. (www.sistemasgenomicos.com), Spain.

Assembly of the complete $p 47$ gene sequences was carried out using CAP3 software (Huang \& Madan, 1999). Alignments of the $p 47$ gene sequences and the deduced amino acid sequences were done using CLUSTAL_X (version 1.81). Phylogenetic analyses were carried out using TREECON (version 3.1b) using the Kimura model and the neighbour-joining method (Van de Peer \& De Wachter, 1994). Substitutions (synonymous and non-synonymous), types of site and $p$ distances ( $p S$ and $p \mathrm{~N}$ ) were calculated by the Nei-Gojobori method, whereas overall mean distances and group distances were computed using the Kimura two-parameter model, using MEGA3 (version 3.1) (Kumar et al., 2004). Fingerprints of multiple amino acid sequence 
Table 1. Primers used for detection (D) and sequencing (F) of the $p 47$ gene

\begin{tabular}{|c|c|c|c|c|c|}
\hline Primer & Sequence $\left(5^{\prime} \rightarrow 3^{\prime}\right)$ & Location $(\mathbf{n t})^{*}$ & $\begin{array}{c}\text { Annealing } \\
\text { temperature }\left({ }^{\circ} \mathrm{C}\right)\end{array}$ & Product (bp) & Strain \\
\hline D-L & GTTGCCGGGCGAATACGAAATGACTT & $322-346$ & 67 & 514 & All \\
\hline $\mathrm{D}-\mathrm{R}$ & GACGCGCCGCCGACCACCTT & $833-814$ & & & \\
\hline F1-L & GTGTGCTGCTCGCGCGATTTTTC & $<146-<124$ & 60 & 380 & All \\
\hline F1-R & GCTTGCGGCCGCTGTTGTTTTTA & $232-210$ & & & \\
\hline F2-L & AGTATCGCCGTCAACGACA & $138-156$ & 61 & 228 & All \\
\hline F2-R & ATTGGTCAAAAGACCGCAAG & $365-346$ & & & \\
\hline F3-L & GCCGCAAGCTCGAATGGGAAATC & $225-247$ & $68 \dagger$ & 228 & All \\
\hline F3-R & CGGCGAGCGGTTGGGACAGTT & $453-433$ & & & \\
\hline F4-L & TTGCCGGGCGAATACGAAATGACT & $322-345$ & 68 & 512 & All \\
\hline $\mathrm{F} 4-\mathrm{R}$ & GACGCGCCGCCGACCAC & $833-817$ & & & \\
\hline F5A-L & AAAGATGCCGGRTTTACYG & 664-682 & 56 & 993 & GLD, M7, NMB, Nm16, Nm22, \\
\hline F5A-R & AGGCGCACATAATCGAAAA & $>489->471$ & & & $\mathrm{Nm} 68, \mathrm{NmP} 17$ \\
\hline F5B-L & GAAGGAAATTGCAGCGAAAC & 737-756 & 58 & 513 & Nm62, Nm63, NmP0, NmP2, \\
\hline F5B-R & GCAATGCCGTACTGGTTTTT & $>82->63$ & & & $\begin{array}{l}\text { NmP3, NmP5, NmP7, NmP18, } \\
\text { NmP19 }\end{array}$ \\
\hline F5B-L & GAAGGAAATTGCAGCGAAAC & $737-756$ & 58 & 638 & Nm59, Nm60, Nm70 \\
\hline F5C-R & ATTAACCGATAGCGGCAGGT & $>207->188$ & & & \\
\hline F5D-L & AGCTTTTGARCGAACTGCAC & $602-621$ & 63 & 1304 & B16b6, Nm9, Nm17, Nm20, \\
\hline F5D-R & GCGGTGTGTTTGATGATGTY & $>738->719$ & & & $\begin{array}{l}\text { Nm21, Nm30, Nm39, Nm40, } \\
\text { NlP3, NlP5, NlP6, NlP9 }\end{array}$ \\
\hline
\end{tabular}

${ }^{*}<$, Position upstream of the $p 47$ gene; $>$, position downstream of the $p 47$ gene. $\dagger 64{ }^{\circ} \mathrm{C}$ for NmP7.

alignments were produced using the alignment shading software TeXshade (Beitz, 2000).

\section{RESULTS}

\section{Surface expression and accessibility of P47}

Fig. 1 shows the results of the flow cytometry analysis of the reactivity of anti-P47 and anti-OMV rabbit sera with cells from some representative $N$. meningitidis and commensal Neisseria strains. Binding of anti-P47 antibodies was highly variable among $N$. meningitidis strains, but this variability did not occur with anti-OMV antibodies (Fig. 1, top and middle rows). The same variability was observed for the commensal Neisseria strains, with much higher binding values for $N$. lactamica strains, in which anti-P47 antibodies reacted with intensities similar to those seen in many $N$. meningitidis strains (Fig. 1, bottom row). Quantitative expression of P47 on the cell surface of all strains tested is shown in Fig. 2. Results shown are the means of at least two assays carried out on different days. Fluorescence intensity was widely variable among strains, although the mean for all strains was higher in $N$. meningitidis. As described above, the reactivity of some single commensal Neisseria was comparable to that of some N. meningitidis strains.

Flow cytometry was also used to evaluate the surface accessibility in some selected strains after permeabilization with ethanol (Fig. 3). Permeabilization increased the accessibility of P47 to specific antibodies $(P<0.05)$ in four of the seven $N$. meningitidis strains, whereas it decreased accessibility in the other three $N$. meningitidis $(P<0.05)$ and in both $N$. lactamica strains (not statistically significant). Reactivity with anti-OMV serum after permeabilization was also variable, although the differences between permeabilized and non-permeabilized cells were much less significant (results not shown).

Differences in surface accessibility of P47 in the strain H44/ 76 and three homologous LOS-deficient mutants ( $p x A$, $r f a C$ and galE strains) are shown in Fig. 4. Binding of antiP47 antibodies to the wild-type strain H44/76 was low, but was significantly increased in the three mutants (see also Fig. 2).

\section{DNA sequencing and phylogenetic analysis}

One possible explanation for apparent strain differences in reactivity with the anti-P47 antisera in flow cytometry is polymorphisms in the $\mathrm{P} 47$ protein. To test this possibility, we sequenced the $p 47$ gene. PCR amplifications using the $\mathrm{D}-\mathrm{L} / \mathrm{D}-\mathrm{R}$ primer pair demonstrated that the $p 47$ gene was present in all strains analysed. Sequencing of the entire gene was carried out by amplification of five overlapping fragments using the F1 to F5 primer pairs shown in Table 1. Amplification of the fifth fragment required four different primer pairs, depending on the strain, due to the existence 


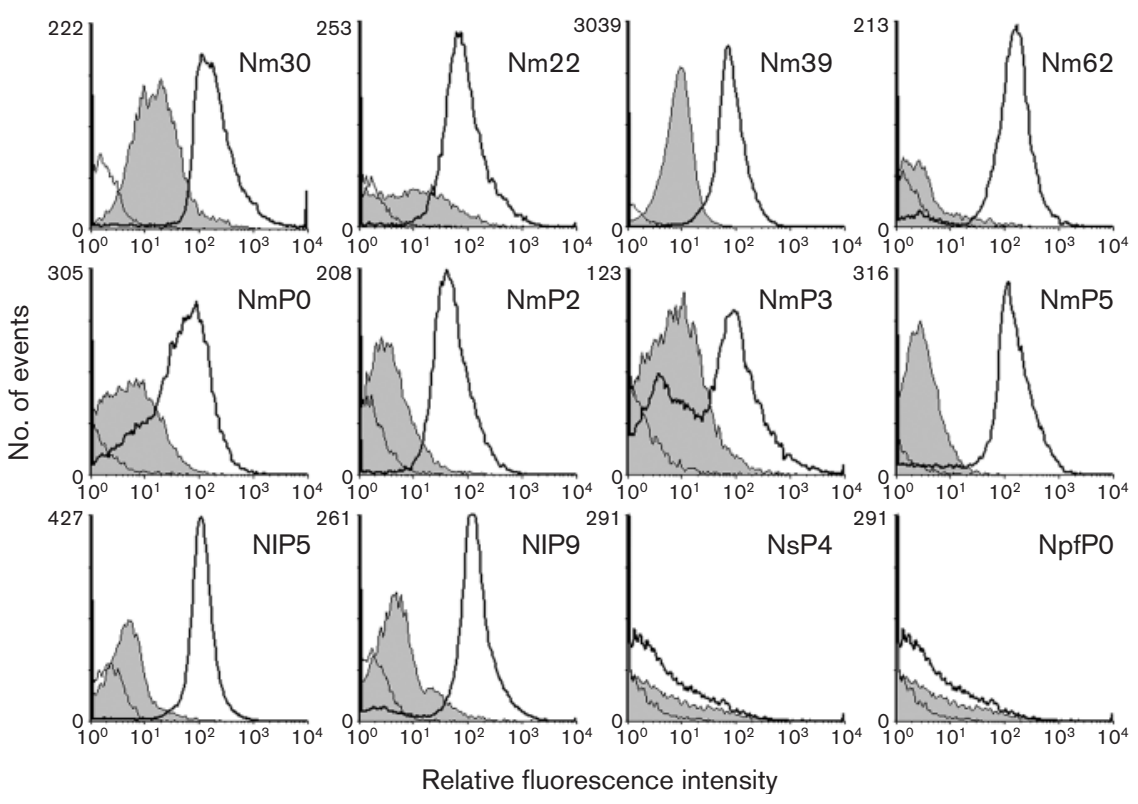

Fig. 1. Flow cytometry analysis of the reactivity of anti-P47 (filled peaks) and anti-OMV (peaks with thick lines) rabbit sera with formalin-treated cells from representative $N$. meningitidis and commensal Neisseria strains. Reactivity with a non-immune rabbit serum (peaks with thin lines) was used as a negative control.

of a hypervariable non-coding region between the annotated NMB0035 ( $p 47)$ and NMB0036 loci. The assembled gene had the same length in all strains (1167 bp), showing only single nucleotide substitutions with no insertions, deletions or intragenic codon stops, and coding for 13 different amino acid sequences (Fig. 5). The overall mean distance, type and number of substitutions, and potential site and $p$ distances, as well as ratios for synonymous and non-synonymous substitutions, are shown in Table 2. Mean distances between groups are shown in the inset of Fig. 5 .

To perform phylogenetic analyses, the registered sequences of the homologous genes from strains $N$. meningitidis Z2491, N. meningitidis MC58, Neisseria gonorrhoeae FA1090 and N. lactamica NISANGER (currently being sequenced at the Sanger Institute) were also included.
The degree of similarity between the amino acid sequences derived from alignment analysis ranged from 97.4 to $99.7 \%$ for N. meningitidis and from 97.7 to $99.0 \%$ for $N$. lactamica. The sequence of the protein from the $N$. gonorrhoeae strain analysed showed a $95.4 \%$ similarity to those of the most divergent N. meningitidis strains. Fig. 6 shows an alignment fingerprint of the 13 amino acid sequence types.

\section{DISCUSSION}

Development of vaccines effective against serogroup B meningococci is one of the major challenges for researchers in this field. Antigens suitable for effective vaccines should be highly conserved, easily accessible at the bacterial surface

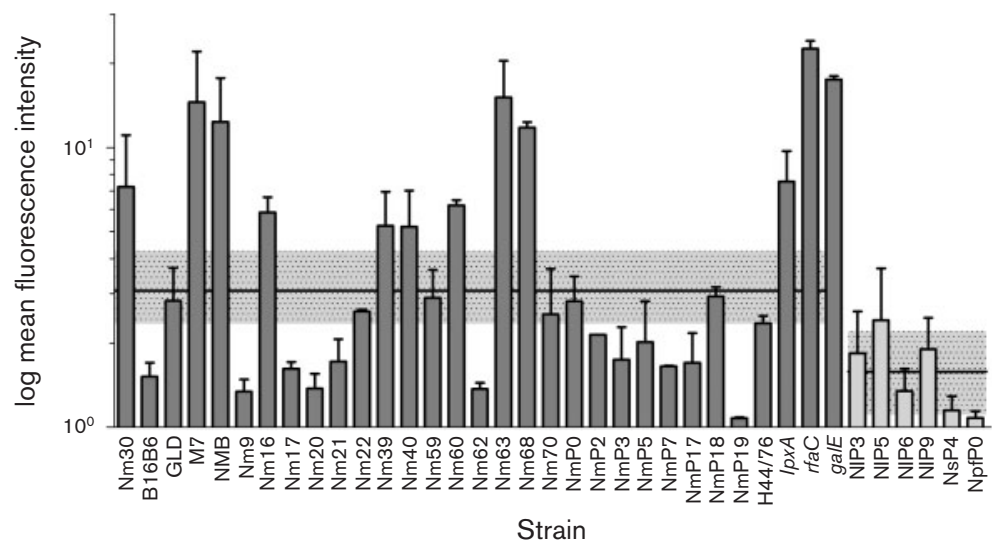

Fig. 2. Interstrain variability of the reactivity of anti-P47 rabbit serum with formalin-treated cells, measured by quantitative flow cytometry. Bars represent the mean fluorescence intensity ( \pm SEM) of all measured cells in a single strain (dark grey bars, N. meningitidis; light grey bars, commensal Neisseria). Lines indicate the geometric mean for each group ( $N$. meningitidis or commensal Neisseria) and shaded boxes show the $95 \%$ confidence interval of the means. 


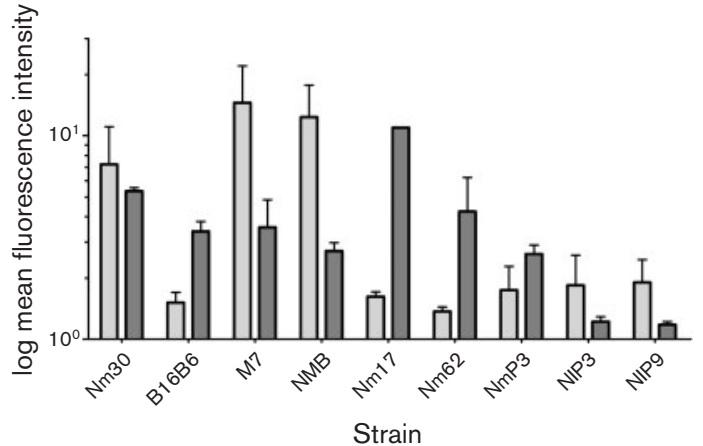

Fig. 3. Reactivity of anti-P47 rabbit serum with formalin-treated cells non-permeabilized (light grey bars) or permeabilized with ethanol (dark grey bars), measured by quantitative flow cytometry. Bars represent the mean fluorescence intensity ( \pm SEM) of all measured cells in a single strain.

and able to elicit long-lasting bactericidal responses. Currently, the most promising approaches are based on OMPs, which are being investigated using several strategies, including reverse vaccinology (Adu-Bobie et al., 2003), heterologous commensal strains (Gorringe et al.,

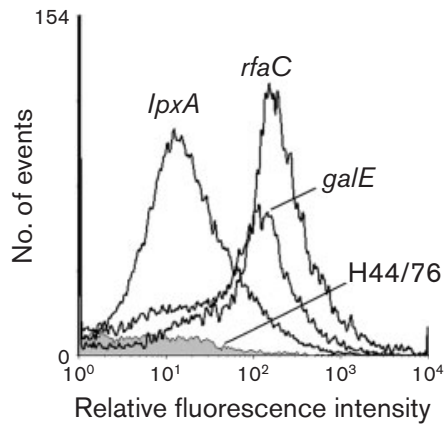

Fig. 4. Reactivity of anti-P47 rabbit serum with formalin-treated cells obtained from strain $\mathrm{H} 44 / 76$ (LOS, lipid A-KDO $2-\mathrm{Hep}_{2}$-Glc$\mathrm{GlcNAc}_{2}-\mathrm{Gal}_{2}$; shaded profile) and the LOS-deficient mutants IpxA (complete lack of LOS), rfaC (LOS, lipid A-KDO ${ }_{2}$ ) and galE (LOS, lipid A- $\mathrm{KDO}_{2}-\mathrm{Hep}_{2}$-Glc-GlcNAc), measured by quantitative flow cytometry.

2005) and multiple PorA expression mutants (Martin et al., 2000). In previous studies, we found a $47 \mathrm{kDa}$ meningococcal OMP inducible by iron restriction, which showed high antigenic conservation among strains and the ability to induce bactericidal responses in vitro (Arenas et al., 2006).

\begin{tabular}{|l|c|c|}
\hline & {$[1]$} & {$[2]$} \\
\hline [1] N. meningitidis & & \\
\hline [2] N. lactamica & 0.024 & \\
\hline [3] N. gonorrhoeae & 0.040 & 0.030 \\
\hline
\end{tabular}

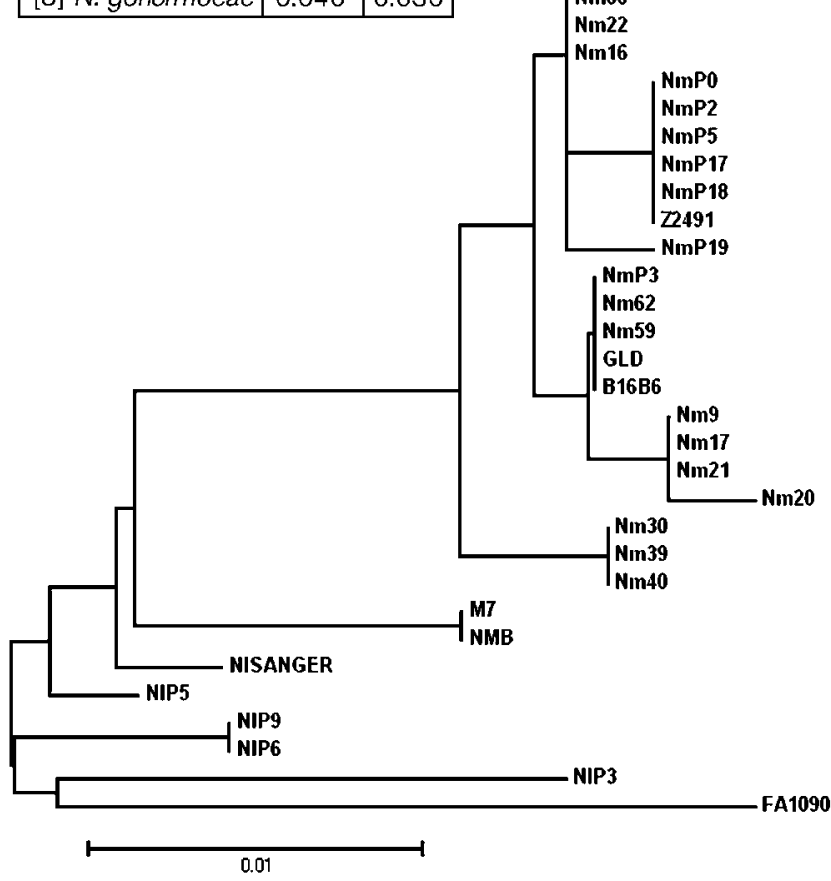

II

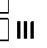
sequence variants

$\mathbf{X}$

$\mathbf{X I}$

XII

XIII

Fig. 5. Neighbour-joining phylogenetic tree of the deduced amino acid sequences of the P47 protein. The sequence of the $N$. gonorrhoeae strain (most divergent) was used to root the tree. Thirteen sequence types were identified, eight in N. meningitidis, four in N. lactamica and one in N. gonorrhoeae. Mean distances between groups are shown in the inset. 
Table 2. Overall mean distances, synonymous and non-synonymous substitutions and sites, $p$ distances of substitutions and $p S / p N$ ratio in the $p 47$ gene

\begin{tabular}{|c|c|c|c|c|c|c|c|}
\hline \multirow[t]{2}{*}{ Species } & \multirow{2}{*}{$\begin{array}{l}\text { No. of amino acid } \\
\text { sequence types }\end{array}$} & \multirow{2}{*}{$\begin{array}{l}\text { Overall mean } \\
\text { distance }\end{array}$} & \multicolumn{5}{|c|}{ Substitution } \\
\hline & & & Type & Number & Potential sites & $p$ distance $(p S$ or $p \mathrm{~N})$ & $p \mathrm{~S} / p \mathrm{~N}^{*}$ \\
\hline N. meningitidis & & & Non-synonymous & 3 & 894 & 0.003 & \\
\hline \multirow[t]{2}{*}{ N. lactamica } & 4 & 0.037 & Synonymous & 37 & 269 & 0.139 & 23.2 \\
\hline & & & Non-synonymous & 6 & 895 & 0.006 & \\
\hline
\end{tabular}

${ }^{*} \mathrm{~A} p \mathrm{~S} / \mathrm{pN}$ ratio of $<1$ indicates a positive selection for amino acid replacement; ratios $>1$ indicate a negative selection, suggesting that conservation of the protein sequence is critical and only neutral changes at the amino acid sequence level are tolerated.

Flow cytometry results obtained with formalin-treated cells were comparable to those using live intact cells (not shown) and demonstrated the accessibility of P47 at the bacterial surface. As can be seen in Figs 1 and 2, the antiP47 mean binding intensity was variable, providing evidence for significant differences among the N. meningitidis strains, and between these and the commensal Neisseria tested. These differences could be due to variability in expression, surface accessibility or P47 epitope arrangements in the different strains. Previous results obtained by Western blotting (in which anti-P47 serum reacted with linear epitopes) showed no differences between meningococcal strains, suggesting that the interstrain variability observed by flow cytometry was not due to differential protein expression, but was probably caused by differences in the accessibility and/or epitope arrangement of the P47.

Antigen masking by surface structures, such as the capsule and lipopolysaccharides, has already been demonstrated. Ethanol damages bacterial outer structures, partially eliminating the capsule and LOS outer residues, exposing surface antigens and facilitating their accessibility to antibodies (Jolley et al., 2001; Michaelsen et al., 2001). In $N$. meningitidis, capsular structure and/or the amount and length of LOS can significantly vary among strains, and all commensal Neisseria lack a capsule and have particular LOS due to the absence of lgt genes (which encode proteins involved in glycosyltransferase synthesis) in most species (Zhu et al., 2002; Kahler et al., 2005).

Thus, we checked the possibility of steric hindrance of the binding of anti-P47 antibodies by capsules or LOS by comparing ethanol-treated and untreated cells for a capsulated strain (NMB) and its non-capsulated mutant (M7), and a wild-type strain (H44/76) and three homologous LOS mutants, one without any LOS and the other two with LOS carrying a truncated oligosaccharide chain (lpxA completely lacks LOS, $r f a C$ has only the lipid A molecule and two KDO residues, and galE has lipid A and two KDO residues linked to two heptoses, glucose and $\mathrm{N}$ acetylglucosamine). Our results did not show congruent differences between ethanol-treated and untreated cells, as some strains significantly increased binding of anti-P47

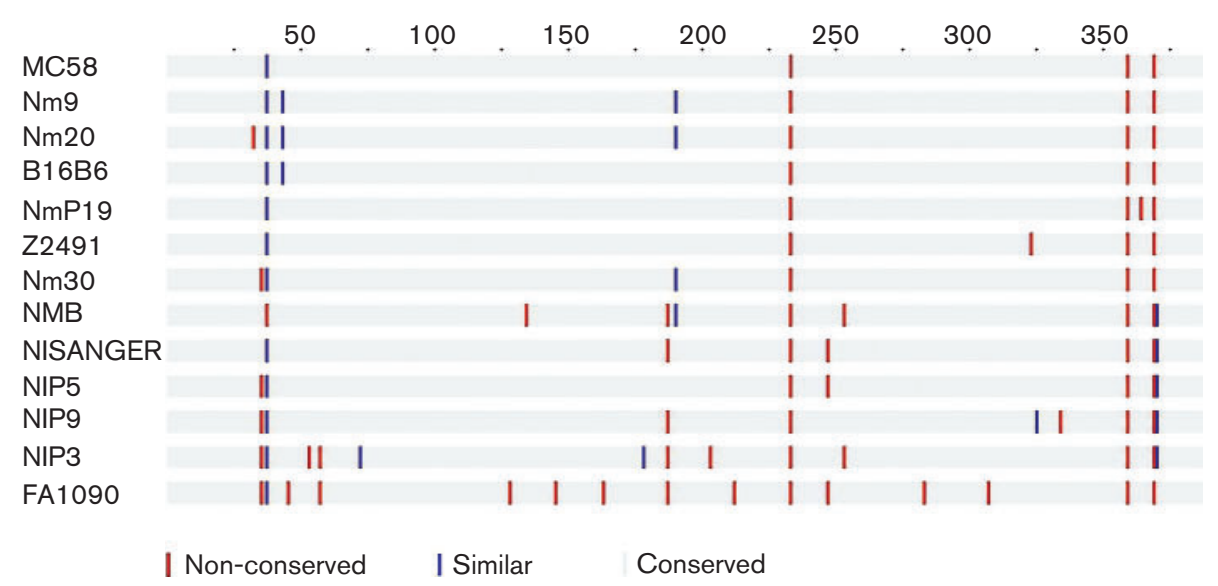

Fig. 6. Alignment fingerprint of the 13 amino acid sequence types produced using the shading software TeXshade. Nonconserved, similar and conserved residues are indicated. 
antibodies after treatment, whereas others showed the opposite effect. It is noteworthy that ethanol produced a significant diminution of binding after treatment in most of the strains that showed the higher binding of anti-P47 antibodies by untreated cells. It is possible that, in these cases, ethanol could affect the P47 epitope structure due to a more exposed conformation.

In contrast, binding to the LOS mutants was significantly higher than to the homologous wild-type strain. It is noteworthy that binding to the lpxA strain, which completely lacks LOS, was lower than that to the $r f a C$ and galE strains with incomplete LOS molecules (Gorter et al., 2003). It is possible that the presence of LOS molecules, even incomplete, could affect the correct composition of the whole outer membrane or the location of some of their protein components. Previous studies on the proteome of wild-type and LOS-deficient OMVs in fact point to differences in their protein composition (Williams et al., 2007). In addition, the iron-regulated lipoproteins TbpB and LbpB have been found in much lower amounts in outer membranes of the LPS-deficient lpxA mutant (Steeghs et al., 2001). As P47 is also an iron-regulated lipoprotein, its expression may be similarly reduced in the lpxA mutant.

Other authors have not noted an effect of capsular material on surface-antigen accessibility (Bowden et al., 1995), which, together with our results, strongly point to LOS as being responsible for the differences in accessibility of P47 to antibodies. The observed variability among strains and the opposing effects of ethanol treatment could be explained by the significant differences in LOS structure and variability found in Neisseria, even among cells in a single strain population (Zhu et al., 2002; Kahler et al., 2005).

The information obtained by analysis of the nucleotide and/or amino acid sequence data using bioinformatic tools can be useful to predict some characteristics of the molecules and to help in the selection of the most promising antigenic variants. Specialized web-based servers and analysis software allow a statistical prediction of characteristics, such as cellular location, hydrophobicity, secondary and tertiary structure, and potential epitopes (Zhang et al., 1998; Yu et al., 2002).

Sequencing of the $p 47$ gene was done using five primer pairs due to the poor results obtained with primer pairs covering longer fragments. Genetic analyses demonstrated 23 alleles for the $p 47$ gene, giving rise to 13 amino acid sequences, with an apparent correlation (although not complete) between some allele types and the clonal complexes assigned to the strains (i.e. alleles 1 and 2 with complex ST-32, and alleles 11-16 with complex ST-8). It is noteworthy that the allele carried by the $N$. lactamica NISANGER strain is closer to those of the $N$. meningitidis strains (particularly to strain NMB and its capsule-deficient mutant M7) than to those of the other N. lactamica strains analysed. The amino acid sequence of the P47 protein showed a $90 \%$ similarity to COG2822 (a predicted periplasmic lipoprotein involved in iron transport in Escherichia coli, Yersinia pestis, Staphylococcus aureus, Bacillus subtilis and Listeria innocua) and a $65 \%$ similarity with DUF451 (predicted putative lipoprotein in E. coli, $S$. aureus and B. subtilis), which is in agreement with the ironregulated character of P47. Predictions also showed a high probability that the molecule is located in the outer membrane $(P=0.79)$ and a very low probability $(P=0.06)$ that it is located in the periplasm.

We believe that differences in reactivity with the anti-P47 antisera in flow cytometry are not due to polymorphisms in the P47 protein because, although 13 amino acid sequence types were demonstrated, the percentage of nonsynonymous substitutions found was low, and the very high $p \mathrm{~S} / \mathrm{pN}$ ratios indicated that the sequence could be considered extremely conserved (Table 2). The results obtained in this work confirm the antigenic cross-reactivity of P47 in all N. meningitidis strains and some commensal Neisseria, particularly $N$. lactamica, and demonstrate its surface location. Correlation of the sequence types with antibody binding by flow cytometry was not significant. Thus, the differences observed are likely to be due to masking by LOS or other surface molecules, or to association of P47 forming protein complexes in which conformational and/or shared epitopes could be dominant. This possibility is currently being investigated in depth. Our results suggest that masking by, or association with, LOS or other membrane molecules can be crucial for antigen accessibility, which must be thoroughly analysed in new vaccine candidates.

\section{ACKNOWLEDGEMENTS}

We wish to thank Drs Peter Borriello, Dan Granoff and Mariagrazia Pizza for kindly providing some of the strains used in this study. This work was supported by grants PGIDT01BIO20301PR from the Xunta de Galicia and PI050178 from the Fondo de Investigación Sanitaria (Ministerio de Sanidad y Consumo), Spain.

\section{REFERENCES}

Adu-Bobie, J., Capecchi, B., Senisto, D., Rappouli, R. \& Pizza, M. (2003). Two years into reverse vaccinology. Vaccine 21, 605-610.

Arenas, J. A., Abel, A., Sánchez, S., Alcalá, B., Criado, M. T. \& Ferreirós, C. M. (2006). Locus NMB0035 codes for a $47 \mathrm{kDa}$ surfaceaccessible conserved antigen in Neisseria. Int Microbiol 9, 273-280.

Beitz, E. (2000). TeXshade: shading and labeling of multiple sequence alignment using LaTeX2e. Bioinformatics 16, 135-139.

Bowden, R. A., Cloeckaert, A., Zygmunt, M. S., Bernard, S. \& Dubray, G. (1995). Surface exposure of outer membrane protein and lipopolysaccharide epitopes in Brucella species studied by enzyme-linked immunosorbent assay and flow cytometry. Infect Immun 63, 3945-3952.

Doytchinova, I. A. \& Flower, D. R. (2002). Quantitative approaches to computational vaccinology. Immunol Cell Biol 80, 270-279.

Gorringe, A. R., Halliwell, D., Matheson, M., Reddin, K., Finney, M. \& Hudson, M. (2005). The development of a meningococcal disease 
vaccine based on Neisseria lactamica outer membrane vesicles. Vaccine 23, 2210-2213.

Gorter, A. D., Oostrikb, J., van der Ley, P., Hiemstrac, P. S., Dankerta, J. \& van Alphen, L. (2003). Involvement of lipooligosaccharides of Haemophilus influenzae and Neisseria meningitidis in defensinenhanced bacterial adherence to epithelial cells. Microb Pathog 34, $121-130$.

Huang, X. \& Madan, A. (1999). CAP3: a DNA sequence assembly program. Genome Res 9, 868-877.

Jolley, K. A., Appleby, L., Wright, J. C., Christoloulides, M. \& Heckels, J. E. (2001). Immunization with recombinant Opc outer membrane protein from Neisseria meningitidis: influence of sequence variation and levels of expression on the bactericidal immune response against meningococci. Infect Immun 69, 3809-3816.

Kahler, C. M., Datta, A., Tzeng, Y. L., Carlson, R. W. \& Stephens, D. S. (2005). Inner core assembly and structure of the lipooligosaccharide of Neisseria meningitidis: capacity of strain NMB to express all known immunotype epitopes. Glycobiology 15, 409-419.

Khan, A. S., Mujer, C. V., Alefantis, T. G., Connolly, J. P., Mayr, V. B., Walcher, P., Lubilz, W. \& DelVecchio, U. G. (2006). Proteomics and bioinformatics strategies to design countermeasures against infectious threat agents. J Chem Inf Model 46, 111-115.

Kumar, S., Tamura, K. \& Nei, M. (2004). MEGA3: integrated software for molecular evolutionary genetics analysis and sequence alignment. Brief Bioinform 5, 150-163.

Leclerc, C. L. (2003). New approaches in vaccine development. Comp Immunol Microbiol Infect Dis 26, 329-341.

Martin, S. L., Borrow, R., van der Ley, P., Dawson, M., Fox, A. J. \& Cartwright, K. A. (2000). Effect of sequence variation in meningococcal PorA outer membrane protein on the effectiveness of a hexavalent PorA outer membrane vesicle vaccine. Vaccine 18, 2476-2481.

Michaelsen, T. E., Aase, A., Kolberg, J., Wedege, E. \& Rosenqvist, E. (2001). PorB3 outer membrane protein on Neisseria meningitidis is poorly accessible for antibody binding on live bacteria. Vaccine 19, 1526-1533.

Morley, S. L. \& Pollard, A. J. (2001). Vaccine prevention of meningococcal disease, coming soon? Vaccine 20, 666-687.

Morley, S. L., Cole, M. J., Ison, C. A., Camaraza, M. A., Sotolongo, M., Anwar, N., Cuevas, I., Carbonero, M., Campa, H. C. \& other authors (2001). Immunogenicity of a serogroup $B$ meningococcal vaccine against multiple Neisseria meningitidis strains in infants. Pediatr Infect Dis J 20, 1054-1061.

O'Dwyer, C. A., Reddin, K., Martin, D., Taylor, S. C., Gorringe, A. R., Hudson, M. J., Brodeur, B. R., Langford, P. R. \& Kroll, J. S. (2004). Expression of heterologous antigens in commensal Neisseria spp.: preservation of conformational epitopes with vaccine potential. Infect Immun 72, 6511-6518.

Petrovsky, N. \& Brusic, V. (2002). Computational immunology: the coming of age. Immunol Cell Biol 80, 248-254.
Pintor, M., Ferrón, L., Gómez, J. A., Powell, N. B. L., Ala'Aldeen, D. A. A., Borrriello, S. P., Criado, M. T. \& Ferreirós, C. M. (1996). Blocking of iron uptake from transferrin by antibodies against the transferrin binding proteins in Neisseria meningitidis. Microb Pathog 20, 127-139.

Rosenqvist, E., Hoiby, E. A., Wedege, E., Bryn, K., Kolberg, J., Klem, A., Ronnild, E., Bjune, G. \& Nokleby, H. (1995). Human antibody responses to meningococcal outer membrane antigens after three doses of the Norwegian group B meningococcal vaccine. Infect Immun 63, 4642-4652.

Steeghs, L., de Cock, H., Evers, E., Zomer, B., Tommassen, J. \& van der Ley, P. (2001). Outer membrane composition of a lipopolysaccharide-deficient Neisseria meningitidis mutant. EMBO J 20, 6937-6945.

Tappero, J. W., Lagos, R., Ballesteros, A. M., Plikaytis, B., Williams, D., Dykes, J., Gheesling, L. L., Carlone, G. M., Hoiby, E. A. \& other authors (1999). Immunogenicity of 2 serogroup B outer-membrane protein meningococcal vaccines: a randomized controlled trial in Chile. JAMA 281, 1520-1527.

Troncoso, G., Sánchez, S., Moreda, M., Criado, M. T. \& Ferreirós, C. M. (2000). Antigenic cross-reactivity between outer membrane proteins of Neisseria meningitidis and commensal Neisseria species. FEMS Immunol Med Microbiol 27, 103-109.

Valdivia, R. H. \& Falkow, S. (1998). Flow cytometry and bacterial pathogenesis. Curr Opin Microbiol 1, 359-363.

Van de Peer, Y. \& De Wachter, R. (1994). TREECON for Windows: a software package for the construction and drawing of evolutionary trees for the Microsoft Windows environment. Comput Appl Biosci 10, 569-570.

Welsch, J. A., Moe, G. R., Rossi, R., Adu-Bobie, J., Rappouli, R. \& Granoff, D. M. (2003). Antibody to genome-derived neisserial antigen 2132, a Neisseria meningitidis candidate vaccine, confers protection against bacteremia in the absence of complement-mediated bactericidal activity. J Infect Dis 188, 1730-1740.

Williams, J. N., Skipp, P. J., Humphries, H. E., Christodoulides, M., O'Connor, C. D. \& Heckels, J. E. (2007). Proteomic analysis of outer membranes and vesicles from wild-type serogroup B Neisseria meningitidis and a lipopolysaccharide-deficient mutant. Infect Immun 75, 1364-1372.

Yu, K., Petrovsky, Y. K., Schönbach, C., Koh, J. Y. \& Brusic, V. (2002). Methods for prediction of peptide binding to MHC molecules: a comparative study. Mol Med 8, 137-148.

Zagursky, R. J. \& Rusell, D. (2001). Bioinformatics: use in bacterial vaccine discovery. Biotechniques 31, 636-640.

Zhang, C., Anderson, A. \& DeLisi, C. (1998). Structural principles that govern the peptide-binding motifs of class I MHC molecules. $\mathrm{J} \mathrm{Mol}$ Biol 281, 929-947.

Zhu, P., Klutch, M. J., Bash, M. C., Tsang, R. S. W., Ng, L. \& Tsai, C. (2002). Genetic diversity of three lgt loci for biosynthesis of lipooligosaccharide (LOS) in Neisseria species. Microbiology 148, 1833-1844. 